• 研究报告・

\title{
非山列岛自然保护区虾类优势种生态位
}

\author{
徐开达 卢衍尔 ${ }^{*}$ 卢占晖 戴 乾
}

(浙江省海洋水产研究所, 浙江舟山 316021)

\begin{abstract}
摘要：根据非山列岛自然保护区2015年11月，2016年2月、5月、8月的调查资料，运用相对重要性指数(index of relative importance, $I R I$ )、生态位宽度以及生态位重叠指数等生态指标, 对调查海域的优势种虾类进行生态位特征 分析，以了解该海域内虾类组成及优势种间的关系。结果表明，保护区共调查到虾类16种，隶属8科12属，均属暖 温种或暖水种, 其中优势种 (IRI > 500)有7种, 隶属于4科5属; 虾类相对资源量呈春秋季高, 夏冬季低的季节性分 布特征。除脊尾白虾(Exopalaemon carinicauda) 外, 优势种的时空生态位宽度值(时间生态位宽度与空间生态位宽 度的乘积)均大于非优势种。日本鼓虾(Alpheus japonicus)和细巧仿对虾(Parapenaeopsis tenella)的时空二维生态位 重叠值最大, 表明其对资源的利用具有相似性; 安氏白虾(Exopalaemon annandalei)和哈氏仿对虾(Parapenaeopsis hardwickii) 的时空生态位重叠值最小, 说明二者相关性低。通过计算得知, 相对重要性指数( IRI)与生态位宽度对物 种重要性排序结果有异: 时空生态位宽度值最大的为葛氏长臂虾(Palaemon gravieri), 而IRI值最高的为日本鼓虾。 与相对重要性指数 $(I R I)$ 相比, 生态位宽度指数不仅能反映物种对资源的利用能力, 还能反映物种分布是否均匀, 但这两者均不能反映生物量的多少。
\end{abstract}

关键词: 非山列岛自然保护区; 虾类组成; 生态位宽度; 生态位重叠; 相对重要性指数

\section{Ecological niche analysis of dominant shrimp species in the Jiushan Islands Marine Nature Reserve}

\author{
Kaida Xu, Kaner Lu*, Zhanhui Lu, Qian Dai \\ Marine Fisheries Research Institute of Zhejiang, Zhoushan, Zhejiang 316021
}

\begin{abstract}
The broad aim of our study was to understand shrimp communities and the relationship between dominant species by conducting ecological niche analyses. We collected data with respect to quantities, biomass and species identification on shrimps from the Jiushan Islands Marine Nature Reserve in November 2015 and again in February, May and August of 2016. Using indices such as the index of relative importance $(I R I)$, niche breadth and niche overlap, we analyzed the ecological niche of dominant shrimp species. Finally, we compared niche breadth to understand the relative resource utilization capacity of different shrimp species in the community and also identify whether shrimp species are evenly distributed across the sampling sites/seasons. We used spatio-temporal niche breadth which is a comprehensive descriptor of resource utilization and the product of temporal and spatial niche breadth. In total, we captured 16 warm-temperate and warm-water species in the marine reserve, belonging to 8 families and 12 genera. Seven dominant shrimp species $(I R I>500)$ were identified, belonging to four families and five genera. Results showed that relative shrimp resources varied with season and further revealed that shrimp biomass in spring and autumn were higher compared to summer and winter. Spatio-temporal niche breadth of all dominant species (except Exopalaemon carinicauda) was higher than the other species. The spatio-temporal niche overlap value between Alpheus japonicus and Parapenaeopsis tenella was the highest, indicating that these two species had highly similar resources utilization. On the other hand, Exopalaemon annandalei and Parapenaeopsis hardwickii, were least similar in their resource utilization. The importance ranking results calculated using IRI and niche breadth were not identical: Palaemon gravieri had the highest spatio-temporal niche breadth value, yet Alpheus japonicus had the highest IRI value. We note however that the indices used cannot reflect
\end{abstract}

收稿日期: 2017-12-28; 接受日期: 2018-03-07

基金项目: 国家自然科学基金(31702346)和浙江省科技计划项目(2016F10003, 2016F30018, 2017C32031)

* 通讯作者 Author for correspondence. E-mail: kanerpersonal@foxmail.com 
biomass of the species.

Key words: Jiushan Islands Marine Nature Reserve; shrimp composition; niche breadth; niche overlap; index of relative importance (IRI)

生态位(niche)作为现代生态学的重要理论之一, 已广泛应用于海洋生态系统的研究(徐晓群等, 2013; 彭松耀等, 2015; 汤雁滨等, 2016)。由于同一生境中 的资源有限，不同虾类通常会发生摄食习性、空间 以及时间生态位的分化, 从而提高资源的利用率来 实现共生(韩东燕等, 2013)。与多样性指数、种类丰 富度指数、均匀度指数等生物多样性指数相比, 生 态位分析(如生态位宽度和生态位重叠)不仅能反映 物种对资源的利用能力(张桂莲和张金屯, 2002), 同 时也能表明研究物种间的竞争以及物种对环境的 适应能力(徐治国等, 2007), 还能从一定程度上反映 物种分布的均匀程度(徐春燕等, 2012)。虾类的竞争 与共存同时受时间、空间这两个维度的影响, 可用 这两个维度生态位特征值的乘积来表示二维生态 位的特征值(May, 1975), 用于更加准确地描述虾类 的竞争共存机制(李锐等, 2007)。

非山列岛国家级海洋生态自然保护区于 2003 年成立, 总面积约114,950 ha (张亚洲和贺舟挺, 2013), 是诸多经济鱼类和虾蟹类的产卵场与索饵 场之一(张洪亮等, 2008)。韭山列岛自然保护区是一 个受到严格保护的自然生态系统, 其核心区内严禁 任何生产性的活动。该保护区位于浙江中北部海域, 北邻舟山渔场, 年均表层水温 $10.3^{\circ} \mathrm{C}$, 年均表层盐 度为28.3 (贺舟挺等, 2012)。由于保护区的特性及所 属的地理位置, 对该海域的研究可从一定程度上反 映浙江近岸海域的资源状况。浙江近岸经济虾类多 达20余种(宋海棠等, 2012), 作为重要的渔获资源, 虾类种类及数量的变化会影响整个海洋生态系统 的动态平衡(夏陆军等, 2016), 因而具有重要的研究 意义。此前针对非山列岛海域的相关研究, 仅见部 分学者(张洪亮等, 2008; 贺舟挺等, 2012; 张亚洲和 贺舟挺, 2013; 孙立辉等, 2014)关于渔业资源密度 的论述。为此, 本研究根据2015-2016年四个航次在 非山列岛附近海域开展的渔业资源调查所获得的 虾类调查资料, 分析该海域内虾类的组成、优势种 以及资源量的季节性分布情况, 采用相对重要性指 数确定优势种虾类, 并计算每个优势种的生态位宽 度及不同优势种间的生态位重叠, 重点研究该区域
内虾类优势种资源的现状及特点。

\section{材料与方法}

\section{1 数据来源}

本研究在非山列岛自然保护区核心区海域设 置 4 个断面共 16 个站位 (图 1)。调查范围为 $29^{\circ} 20^{\prime}-29^{\circ} 30^{\prime} \mathrm{N}, 122^{\circ} 08^{\prime}-122^{\circ} 18^{\prime} \mathrm{E}$, 并于 2015 年 11 月(秋)、2016年2月(冬)、5月(春)和8月(夏)开展了 4 个航次的调查。调查船选用单船底拖网渔船, 船长 $38 \mathrm{~m}$, 主机功率 $220 \mathrm{kw}$, 总吨位 $150 \mathrm{t}$ 。调查网具网 口拉紧周长 $50 \mathrm{~m}$, 囊网网目尺寸 $25 \mathrm{~mm}$, 上纲长 $30 \mathrm{~m}$, 下纲长 $38 \mathrm{~m}$ 。调查时, 平均拖速为 $3 \mathrm{kn} / \mathrm{h}$, 每 个站位拖网时间均标准化为 $1 \mathrm{~h}$ 。对每个站位渔获物 随机采样 1 箱 $(20 \mathrm{~kg})$, 参照 《中国海洋生物名录》(刘 瑞玉, 2008)将渔获物中的虾类鉴定至种, 并记录尾 数与质量。调查及测定均严格按照海洋调查规范(中 华人民共和国国家质量监督检验检疫总局, 2007)的 相关规定执行。

\section{2 数据处理}

虾类相对资源量用各站位每小时渔获虾类的 平均质量 $(\mathrm{g} / \mathrm{h})$ 与平均尾数(ind./h)表示。

根据虾类对水温的适应能力, 可将其分为暖水 种、暖温种、冷温种和冷水种(田明诚等, 1993)。

采用相对重要性指数(index of relative importance, IRI)确定优势种(Pinkas et al, 1971), 公式如下:

$$
I R I=[(N+W) \times F] \times 10^{5}
$$

式中, $N$ 为某一物种尾数占比, $W$ 为该物种的重量百 分比, $F$ 为该物种在所有站位中的出现频率百分比。 本研究将IRI > 500的种类定为优势种(卢占晖等, 2015)。

生态位宽度的计算公式(Magurran, 1988):

$$
B_{\mathrm{i}}=-\sum_{i=1}^{S}\left(P_{\mathrm{ij}} \ln P_{\mathrm{ij}}\right)
$$

空间生态位重叠指数的计算公式(Pianka, 1973):

$$
O_{\mathrm{ik}}=\sum_{j=1}^{S}\left(P_{\mathrm{ij}} P_{\mathrm{kj}}\right) / \sqrt{\sum_{j=1}^{S} P_{\mathrm{ij}}^{2} P_{\mathrm{kj}}^{2}}
$$

式中, $P_{\mathrm{ij}}$ 和 $P_{\mathrm{kj}}$ 分别代表第 $\mathrm{j}$ 个站位种 $i$ 和种 $k$ 个体数的 


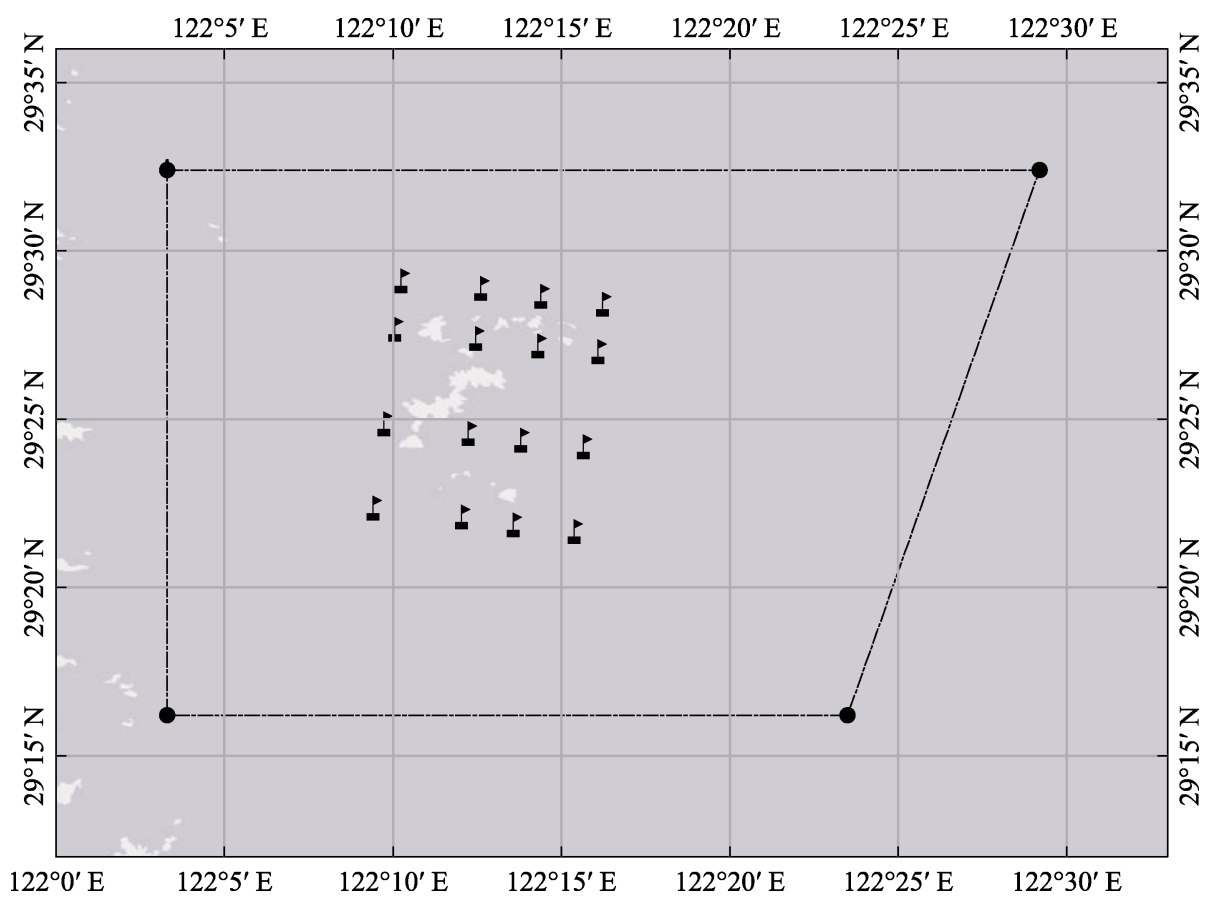

图1 研究海域及采样站位示意图(采样站位设置在保护区核心范围附近)

Fig. 1 Illustration of the investigated area and sampling sites (sampling sites were set around the core area of the Jiushan Islands Marine Nature Reserve)

比例, $S$ 为调查站位总数。当分析空间生态位时, $B_{\mathrm{i}}$ 表示种 $i$ 的空间生态位宽度, $O_{\mathrm{ik}}$ 代表种 $i$ 与种 $k$ 的空间 生态位重叠值(0-1), 其值越大表示两物种对环境资 源的利用越相似; 当分析时间生态位时, $S$ 为调查的 次数, 此时 $B_{\mathrm{i}}$ 表示种 $i$ 的时间生态位宽度, $O_{\mathrm{ik}}$ 代表种 $i$ 与种 $k$ 的时间生态位重叠值, 其反映了不同物种在 时间维度上对资源利用的相似程度(郭天宇和许荣 满, 2003), $B_{\mathrm{i}}$ 的值越大, 表示物种对环境资源的利用 率越高。时空二维生态位宽度值和时空二维生态位 重叠值的计算分别为相应的时间和空间生态位宽 度值、生态位重叠值的乘积(于振海等, 2010)。

根据空间生态位宽度值 $\left(B_{\mathrm{i}}\right)$ 的变化范围, 可将 优势种虾类分为: (1)广生态位种 $\left(B_{\mathrm{i}}>4.00\right)$, 其环境 适应能力强、资源利用率高, 分布广泛; (2)中生态 位种 $\left(2.00<B_{\mathrm{i}} \leq 4.00\right)$, 在资源利用及适应性上稍差, 但分布仍较广; (3)窄生态位种 $\left(B_{\mathrm{i}} \leq 2.00\right)$, 其对生境 选择性强, 对环境敏感, 且分布范围有限(卢占晖等, 2018)。

利用ArcGIS 10.2 for Desktop (版本10.2.0.3348) 绘制站位图、利用Microsoft Excel 2016存储原始数 据, 所有数据的分析处理以及制图均在R 3.3.1 (R Core Team, 2016)中完成。

\section{结果}

\section{1 优势种种类组成}

研究海域共调查到虾类16种，隶属于 8 科12属。 其中种类数最多的为对虾科, 共 4 属 5 种, 占总数的 $31.3 \%$; 其次为长臂虾科, 共计 2 属 4 种, 占总数的 $25.0 \%$; 其余若干科的种数均不超过 2 种(表1)。调查 海域全年优势种为日本鼓虾(Alpheus japonicus)、安 氏白虾 (Exopalaemon annandalei)、葛氏长臂虾 (Palaemon gravieri)、哈氏仿对虾(Parapenaeopsis hardwickii)、中华管鞭虾(Solenocera crassicornis)、 细巧仿对虾 (Parapenaeopsis tenella) 和脊尾白虾 (Exopalaemon carinicauda) 等7种。此次调查中, 暖 水种共9种, 占 $56.25 \%$; 暖温种共7种, 占 $43.75 \%$, 且 绝大部分暖温种 $(85.71 \%)$ 属于对虾科与长臂虾科。

\section{2 相对资源量变化}

非山列岛附近海域虾类相对资源量随季节变 化而波动：最高的为秋季(2015年11月)，春季(2016 年5月)次之; 冬季(2016年2月)的相对资源状况处于 较低水平, 较其他季节差异明显(图2)。平均网获质 量的变化范围为883.4-2,882.9 $\mathrm{g} / \mathrm{h}$, 而平均网获尾 数则在556-2,804 ind./h之间波动。平均网获质量与 
表12015-2016年韭山列岛自然保护区调查海域虾类组成

Table 1 Shrimp composition of Jiushan Islands Marine Nature Reserve in 2015-2016

\begin{tabular}{|c|c|c|c|c|c|}
\hline 科 Family & 属 Genus & 种 Species & $\begin{array}{l}\text { 适温性 } \\
\text { Optimum } \\
\text { temperature }\end{array}$ & $\begin{array}{l}\text { 出现月份 } \\
\text { Occurrence }\end{array}$ & $\begin{array}{l}\text { 相对重要性指数 } \\
\text { Index of relative } \\
\text { importance (IRI) }\end{array}$ \\
\hline \multirow[t]{5}{*}{ 对虾科 Penaeidae } & 新对虾属 Metapenaeus & 周氏新对虾 Metapenaeus joyneri & WW & $\circ \Delta \boldsymbol{\Delta}$ & 99.33 \\
\hline & 明对虾属 Fennerpenaeus & 长毛明对虾 Fenneropenaeus penicillatus & WW & $\circ \Delta \boldsymbol{\Delta}$ & 7.75 \\
\hline & 赤虾属 Metapenaeopsis & 戴氏赤虾 Metapenaeopsis dalei & WT & $\circ \bullet \triangle \Delta$ & 4.62 \\
\hline & 仿对虾属 Parapenaeopsis & 细巧仿对虾 Parapenaeopsis tenella & WT & $\circ \bullet \triangle \Delta$ & $1,002.58$ \\
\hline & & 哈氏仿对虾 Parapenaeopsis hardwickii & WW & $\circ \bullet \Delta \mathbf{\Delta}$ & $2,204.78$ \\
\hline \multirow[t]{4}{*}{ 长臂虾科 Palaemonidae } & 白虾属 Exopalaemon & 安氏白虾 Exopalaemon annandalei & WT & $\circ \bullet \Delta \mathbf{\Delta}$ & $3,944.06$ \\
\hline & & 脊尾白虾 Exopalaemon carinicauda & WT & $\circ \Delta \boldsymbol{\Delta}$ & 752.25 \\
\hline & 长臂虾属 Palaemon & 葛氏长臂虾 Palaemon gravieri & WT & $\circ \bullet \Delta \mathbf{\Delta}$ & $3,008.04$ \\
\hline & & 巨指长臂虾 Palaemon macrodactylus & WT & $\bullet \Delta \Delta$ & 4.15 \\
\hline \multirow[t]{2}{*}{ 鼓虾科 Alpheidae } & 鼓虾属 Alpheus & 日本鼓虾 Alpheus japonicus & WT & $\Delta \boldsymbol{\Delta}$ & $5,370.43$ \\
\hline & & 鲜明鼓虾 Alpheus distinguendus & WW & $\circ \bullet \Delta \boldsymbol{\Delta}$ & 374.41 \\
\hline 藻虾科 Hippolytidae & 鞭腕虾属 Lysmata Risso & 鞭腕虾 Lysmata vittata & WW & $\bullet \boldsymbol{\Delta}$ & 4.4 \\
\hline 樱虾科 Sergestidae & 毛虾属 Acetes & 中国毛虾 Acetes chinensis & WW & $\boldsymbol{\Delta}$ & 123.28 \\
\hline 异指虾科 Processidae & 异指虾属 Processa & 日本异指虾 Processa japonica & WW & $\Delta \boldsymbol{\Delta}$ & 0.89 \\
\hline 管鞭虾科 Solenoceridae & 管鞭虾属 Solenocera & 中华管鞭虾 Solenocera crassicornis & WW & $\bullet \boldsymbol{\Delta}$ & $2,183.67$ \\
\hline 玻璃虾科 Pasiphaeidae & 细螯虾属 Leptochela & 细鳌虾 Leptochela gracilis & WW & $\bullet \boldsymbol{\Delta}$ & 104.97 \\
\hline
\end{tabular}

WW: 暖水性种类; WT: 暖温性种类; ○春; ・夏; $\Delta$ 秋; $\Delta$ 冬

WW, Warm-water species; WT, Warm-temperature species; $\bigcirc$ Spring; • Summer; $\Delta$ Autumn; $\boldsymbol{\Delta}$ Winter

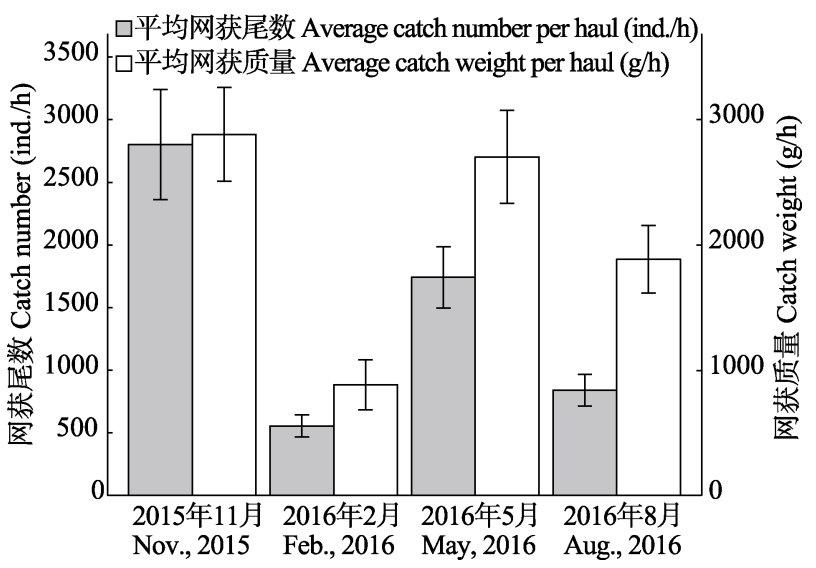

图2 调查海域虾类相对资源量随季节的变化(95\%置信区间) Fig. 2 The change of relative biomass resources of shrimps on a seasonal basis (95\% confidence interval)

平均网获尾数的变化趋势相同, 均呈现出春、秋季 高, 夏、冬季低的波动分布。

\section{3 优势种生态位宽度}

在时间维度上，生态位宽度最高的为葛氏长臂 虾(1.04), 最低的为脊尾白虾(0.31), 哈氏仿对虾、细 巧仿对虾、安氏白虾、日本鼓虾及中华管鞭虾的生 态位宽度值依次递增, 变化范围为 $0.60-0.75$ 。

空间生态位宽度值变化范围为1.40-5.18, 分为:
(1)广生态位种，包括安氏白虾(4.49)、葛氏长臂虾 (4.54)和日本鼓虾(5.18), (2)中生态位种, 包括中华 管鞭虾(3.14)、细巧仿对虾(2.51)及哈氏仿对虾(2.42), (3)以及窄生态位种, 如脊尾白虾(1.40)。

从时空二维生态位宽度来看, 葛氏长臂虾的宽 度值(4.71)最大, 日本鼓虾(3.76)与安氏白虾(3.03) 次之; 而脊尾白虾的宽度值(0.43)最小(表2)。非优势 种中, 仅有鲜明鼓虾(Alpheus distinguendus)的宽度 值(0.49)略高于脊尾白虾, 其余非优势种的宽度值 均远小于优势种。

\section{4 优势种生态位重叠}

在时间维度上，哈氏仿对虾和中华管鞭虾的时 间生态位重叠值(0.99)以及日本鼓虾和细巧仿对虾 的重叠值(0.95)极高, 而安氏白虾和哈氏仿对虾的 重叠值(0.03)最低。21对两两不同的优势种中, 时间 生态位重叠值在 0.6 及以上的有 7 对，占总数的 $33.33 \%$ (表3)。

在空间维度上, 葛氏长臂虾和日本鼓虾 $(0.90)$ 、 哈氏仿对虾和中华管鞭虾 (0.90)以及脊尾白虾和日 本鼓虾(0.89)均具有高空间生态位重叠值, 而脊尾 白虾和细巧仿对虾的重叠值最低(0.51); 其中有13 
对优势种的空间生态位重叠值在 0.6 以上, 占总数 的61.90\% (表3)。

时空二维生态位重叠值的变化范围为 0.02-0.89 (表4); 其中哈氏仿对虾和中华管鞭虾的 时空二维生态位重叠值最高, 为 0.89 , 而安氏白虾 和哈氏仿对虾的时空二维生态位重叠值最低, 仅为 0.02 。

\section{3 讨论}

\section{1 非山列岛海域优势种虾类及其资源量变化}

非山列岛附近海域受江河径流注入、台湾暖流 与黄海暖流交汇等的影响，混合水系丰富(王一鹤 等, 2015), 是诸多广温、广盐性虾类的天然索饵、 产卵场(宋海棠等, 2012), 进而形成了该海域虾类群

表2 优势种的时间、空间以及时空二维生态位宽度对比

Table 2 Comparisons of dominant shrimps among spatial, temporal and spatio-temporal niche breadths

\begin{tabular}{llll}
\hline $\begin{array}{l}\text { Species } \\
\text { Sp种 }\end{array}$ & $\begin{array}{l}\text { 空间生态位宽度 } \\
\text { Spatial niche } \\
\text { breadth }\left(B_{\text {i-space }}\right)\end{array}$ & $\begin{array}{l}\text { 时间生态位宽度 } \\
\text { Temporal niche } \\
\text { breadth }\left(B_{\text {i-time}}\right)\end{array}$ & $\begin{array}{l}\text { 时空二维生态位宽度 } \\
\text { Spatio-temporal } \\
\text { niche breadth }\left(B_{\mathrm{i}}\right)\end{array}$ \\
\hline 安氏白虾 Exopalaemon annandalei & 4.49 & 0.67 & 3.03 \\
葛氏长臂虾 Palaemon gravieri & 4.54 & 1.04 & 4.71 \\
哈氏仿对虾 Parapenaeopsis hardwickii & 2.42 & 0.60 & 1.46 \\
脊尾白虾 Exopalaemon carinicauda & 1.40 & 0.31 & 0.43 \\
日本鼓虾 Alpheus japonicus & 5.18 & 0.73 & 3.76 \\
细巧仿对虾 Parapenaeopsis tenella & 2.51 & 0.64 & 1.61 \\
中华管鞭虾 Solenocera crassicornis & 3.14 & 0.75 & 2.35 \\
\hline
\end{tabular}

表3 韭山列岛自然保护区优势种间空间生态位(对角线以下)和时间生态位(对角线以上)重叠值

Table 3 Spatial niche overlaps (under the diagonal) and temporal niche overlaps (above the diagonal) between dominant shrimp species in the Jiushan Islands Marine Nature Reserve

\begin{tabular}{|c|c|c|c|c|c|c|c|}
\hline & $\begin{array}{l}\text { 安氏白虾 } \\
\text { Exopalaemon } \\
\text { annandalei }\end{array}$ & $\begin{array}{l}\text { 葛氏长臂虾 } \\
\text { Palaemon } \\
\text { gravieri }\end{array}$ & $\begin{array}{l}\text { 哈氏仿对虾 } \\
\text { Parapenaeopsis } \\
\text { hardwickii }\end{array}$ & $\begin{array}{l}\text { 脊尾白虾 } \\
\text { Exopalaemon } \\
\text { carinicauda }\end{array}$ & $\begin{array}{l}\text { 日本鼓虾 } \\
\text { Alpheus } \\
\text { japonicus }\end{array}$ & $\begin{array}{l}\text { 细巧仿对虾 } \\
\text { Parapenaeopsis } \\
\text { tenella }\end{array}$ & $\begin{array}{l}\text { 中华管鞭虾 } \\
\text { Solenocera } \\
\text { crassicornis }\end{array}$ \\
\hline 安氏白虾 Exopalaemon annandalei & & 0.65 & 0.03 & 0.72 & 0.24 & 0.38 & 0.14 \\
\hline 葛氏长臂虾 Palaemon gravieri & 0.67 & & 0.75 & 0.69 & 0.24 & 0.52 & 0.82 \\
\hline 哈氏仿对虾 Parapenaeopsis hardwickii & 0.50 & 0.80 & & 0.13 & 0.11 & 0.39 & 0.99 \\
\hline 脊尾白虾 Exopalaemon carinicauda & 0.54 & 0.85 & 0.53 & & 0.37 & 0.46 & 0.21 \\
\hline 日本鼓虾 Alpheus japonicus & 0.61 & 0.90 & 0.58 & 0.89 & & 0.95 & 0.08 \\
\hline 细巧仿对虾 Parapenaeopsis tenella & 0.65 & 0.72 & 0.58 & 0.51 & 0.77 & & 0.37 \\
\hline 中华管鞭虾 Solenocera crassicornis & 0.58 & 0.83 & 0.90 & 0.65 & 0.71 & 0.56 & \\
\hline
\end{tabular}

加粗数字表示重叠值大于 0.6 , 代表中间重叠水平较高

Values in bold are greater than 0.6, indicating high niche overlaps between two species

表4 优势种间时空二维生态位重叠值

Table 4 Spatio-temporal niche overlap between dominant shrimp species

\begin{tabular}{|c|c|c|c|c|c|c|}
\hline & $\begin{array}{l}\text { 安氏白虾 } \\
\text { Exopalaemon } \\
\text { annandalei }\end{array}$ & $\begin{array}{l}\text { 葛氏长臂虾 } \\
\text { Palaemon } \\
\text { gravieri }\end{array}$ & $\begin{array}{l}\text { 哈氏仿对虾 } \\
\text { Parapenaeopsis } \\
\text { hardwickii }\end{array}$ & $\begin{array}{l}\text { 脊尾白虾 } \\
\text { Exopalaemon } \\
\text { carinicauda }\end{array}$ & $\begin{array}{l}\text { 日本鼓虾 } \\
\text { Alpheus } \\
\text { japonicus }\end{array}$ & $\begin{array}{l}\text { 细巧仿对虾 } \\
\text { Parapenaeopsis } \\
\text { tenella }\end{array}$ \\
\hline 葛氏长臂虾 Palaemon gravieri & 0.43 & & & & & \\
\hline 哈氏仿对虾 Parapenaeopsis hardwickii & 0.02 & 0.60 & & & & \\
\hline 脊尾白虾 Exopalaemon carinicauda & 0.38 & 0.58 & 0.07 & & & \\
\hline 日本鼓虾 Alpheus japonicus & 0.14 & 0.22 & 0.07 & 0.33 & & \\
\hline 细巧仿对虾 Parapenaeopsis tenella & 0.25 & 0.38 & 0.22 & 0.24 & 0.73 & \\
\hline 中华管鞭虾 Solenocera crassicornis & 0.08 & 0.68 & 0.89 & 0.14 & 0.06 & 0.21 \\
\hline
\end{tabular}




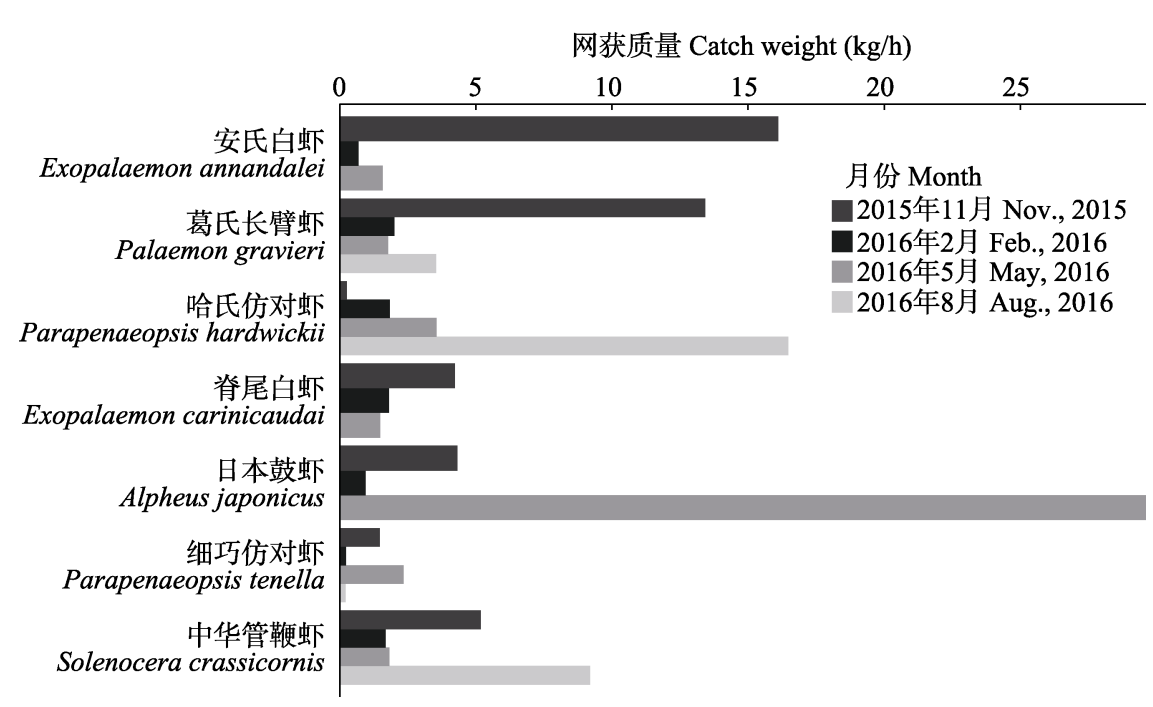

图3 调查区域各季节优势种虾类网获捕获重量变化

Fig. 3 The change of catch weight of dominant shrimp species in each season during the investigation

落结构的多样性。调查海域位于亚热带季风气候区, 太阳辐射较强, 海水温度相对较高(许建平和杨士 英, 1993), 导致暖水种与暖温种占绝对优势。调查 期间捕获的16种虾类均为暖水种或暖温种, 优势种 共7种, 占总种数的 $43.75 \%$ 。其中, 暖水性优势种有 安氏白虾、脊尾白虾、葛氏长臂虾、日本鼓虾及细 巧仿对虾共5种, 占优势种的 $71.43 \%$, 暖温性优势 种有哈氏仿对虾及中华管鞭虾, 占优势种的 $28.57 \%$, 与谢旭等(2017)的研究结果基本一致。

由于虾类的生态群落结构与海洋环境的关系 十分密切(刘瑞玉, 1963), 不同海域优势种虾类不尽 相同。与非山列岛附近海域相比, 东海中部优势种 主要以高温高盐性种类为主, 如假长缝拟对虾 (Parapenaeus fissuroides)、东海红虾 (Plesionika izumiae)、须赤虾(Metapenaeopsis barbata)、长角赤 虾(M. longirostris)和大管鞭虾(Solenocera melantho) 等(卢占晖等, 2015); 而椒江口海域受椒江径流的 影响, 优势种虾类除广温广盐性种类外, 广温低盐 性种类也较多, 如安氏白虾、脊尾白虾、中国毛虾 (Acetes chinensis)、鲜明鼓虾和细螯虾(Leptochela gracilis)等(齐海明等, 2013)。

从调查月份的相对资源量来看, 虾类资源呈春 秋季高, 冬夏季低的分布。冬季 $(2$ 月)资源量很低, 这可能是由于冬季随着水温的下降及暖流影响的 减弱, 导致虾群洄游越冬引起的(齐海明等, 2013; 陈伟峰等, 2017)。在研究海域, 春季是优势种虾类
主要的繁殖季节, 而此时相对资源量主要受到日本 鼓虾的影响(图3), 这可能是由于该物种属于定居性 种类 ${ }^{(1)}$, 且繁殖期较长(宋海棠等, 2012), 其幼体持 续不断地补充到该种群中造成的。从春季到夏季, 长江口径流量逐渐增大, 其携带的营养物质为沿岸 海域浮游植物的繁殖和生长提供了环境基础(唐峰 华等, 2010), 从而为夏季主要优势种之一的哈氏仿 对虾的索伹提供了优越的环境(宋海棠, 2002); 同 时, 长江径流量的增加导致沿岸海域盐度降低, 致 使低盐性种类, 诸如安氏白虾、脊尾白虾和日本鼓 虾, 更有可能选择栖息于低盐的沿岸海域。调查期 间捕获的虾类均属短生命周期的甲壳类生物(宋海 棠等, 2012), 生长较快, 到了秋季幼虾逐渐生长为 成体(齐海明等, 2013), 因而此时相对资源量处于较 高水平。非山列岛自然保护区核心海域每个季节的 虾类资源量受某一虾种的影响极大(图3), 说明调查 海域的优势种虾类随季节变化明显。

\section{2 时间、空间生态位宽度与时空二维生态位宽度}

一个物种的空间生态位宽度值大小代表了该 物种在生境中利用资源的能力, 该物种的分布范围 和生物量则反映了这种能力(张金屯, 2011); 而时间 生态位宽度表示的是不同物种在时间维度上对资 源的利用情况, 从而反映出在研究海域内某物种出 现的时间长短(于振海等, 2010)。

(1) 逢志伟 (2014) 胶州湾虾蟹类群落结构及主要种类渔业生物学 特征. 硕士学位论文, 中国海洋大学, 青岛. 
日本鼓虾、安氏白虾以及葛氏长臂虾均具有高 空间生态位宽度值, 说明这三个物种在调查区域内 分布最广, 且各站位渔获量较为均匀。根据Bennett 等(2010)提出的“不同物种生态位宽度与其生境适应 性密切相关”的结论, 可推测隶属广温广盐性种类的 日本鼓虾与葛氏长臂虾对调查海域的温度、盐度等 环境较适应; 而隶属广温低盐性种类的安氏白虾由 于对其环境适应性较强, 几乎周年均有幼体 (吴常文 和王伟洪, 1995), 从而导致其生态位宽度值也很高。

葛氏长臂虾的时间生态位宽度值最大, 说明其 在调查期间出现的时间跨度最长, 且每个季节的生物 量较为均匀; 该结果与此前的调查结果(贺舟挺等, 2009; 卢占晖等, 2013)相符。而脊尾白虾的时间生 态位宽度值最小, 说明该物种在调查海域的分布具 有季节性, 这主要是由于脊尾白虾的繁殖受季节、水 温的影响(施永海等, 2009), 且夏秋季出生的脊尾白 虾在当年不能繁殖的特性(徐君义, 1990)所引起的。

由于虾类的洄游习性, 其分布同时受到时间和 空间的制约，因而引入时空二维生态位用于更全面 地描述种间关系(李锐等, 2007)。葛氏长臂虾具有最 高的时空生态位宽度值, 表明其各季节分布范围均 较广。

通过比较分析调查海域优势种虾类生态位宽 度可知(表2), 时间、空间以及时空生态位宽度变化 并不完全一致: 具有高空间生态位宽度的物种并不 一定同时具有高时间生态位宽度, 如葛氏长臂虾的 时间生态位宽度值最高, 而其空间生态位宽度值则 小于日本鼓虾。究其原因, 是由于每个物种在时间 和空间维度上对资源的利用能力有所差异造成的 (于振海等, 2010)。

\section{3 时间、空间生态位重叠与时空二维生态位重叠}

生态位宽度表达了物种对资源利用的情况, 而 生态位重叠则揭示了物种间对资源利用的相似性 (李德志等, 2006)。

在时间维度上，中华管鞭虾和哈氏仿对虾拥有 极大的时间生态位重叠值(0.99), 表明在研究海域 这两种虾类对资源的利用随季节变化趋于一致, 这 是由哈氏仿对虾与中华管鞭虾的生长特性所决定 的: 这两种虾类均分布于沿岸低盐水与外海高盐水 交汇的混合水域, 且繁殖期较长, 哈氏仿对虾自 5 月开始繁殖, 7 月至翌年2月均有幼虾出现; 而中华 管鞭虾则自6月开始繁殖, 从9月至翌年1月均有幼
虾出现(宋海棠等, 2009; 薛利建等, 2009)。

在空间维度上，中华管鞭虾与哈式仿对虾的空 间生态位重叠值仍最高, 这也与前文提到的生长特 性有关, 且两者的洄游习性也极为相似: 夏季两种 虾类的幼虾均密集分布于沿岸30 m以浅海域，并在 秋季向外海移动(宋海棠等, 2012)。Wathne等(2000) 研究表明, 当生态位重叠值大于 0.6 时, 种间重叠度 较高。由于葛氏长臂虾的栖息生境和生长特性与哈 氏仿对虾、中华管鞭虾相似，且与这两种虾类的空 间生态位重叠值均在 0.8 以上，从而可据此推测葛 氏长臂虾与日本鼓虾具有高生态位重叠值的原因 可能也与这两种虾类的生存环境与生活习性的相 似性有关。在 21 对两两不同的优势种虾类组中, 有 13 对的空间生态位重叠值高于 0.6 , 占总数的 $61.90 \%$ 。其中，优势种间空间生态位重叠值大于 0.6 的站位重叠率为 $35.49 \%$, 小于 0.6 的站位重叠率为 $23.26 \%$; 而优势种与非优势种间的平均站位重叠率 仅为 $7.47 \%$ 。这印证了葛宝明等(2005)提出的“生态 位重叠值的高低与调查站位中物种间出现的重复 率有关”的结论。

时空二维生态位重叠值为时间与空间生态位 重叠值的乘积，用于更好地衡量种间关系。由于日 本鼓虾和细巧仿对虾在时间与空间维度上的生态 位重叠值(分别为0.77与0.95)均较高, 因而其时空二 维生态位重叠值最大(0.73), 这表明二者的空间同域 性与时间同步性较高(于振海等, 2010)，对资源的利 用趋于一致; 而安氏白虾和哈氏仿对虾的时空二维 生态位重叠值最小(0.02), 说明二者的时间、空间异 质性高, 相关性极低, 几乎不存在共生或竞争关系。

\section{4 生态位宽度与相对重要性指数(IRI)的比较}

相对重要性指数(IRI)被广泛应用于群落结构 研究中, 是用于衡量群落中不同物种优势度最简单 有效的方法。如表2、图4所示，生态位宽度值与相 对重要性指数并无直接关系: 生态位宽度值相对较 高的种类其IRI值不一定高，如葛氏长臂虾的时间 生态位宽度(1.04)与时空二维生态位宽度(4.71)均高 于安氏白虾(分别为 $0.67 、 3.03$ ), 而其IRI值 $(3,008.04)$ 却低于安氏白虾 $(3,944.06)$ 。造成这种差异的主要原 因是这两个指标在计算时考虑的影响因素不同。IRI 值计算时将某一物种的渔获质量占比、尾数占比与 站位出现频率作为衡量依据; 根据Spearman相关分 析和相关性显著分析，某一虾种的IRI值大小与该 


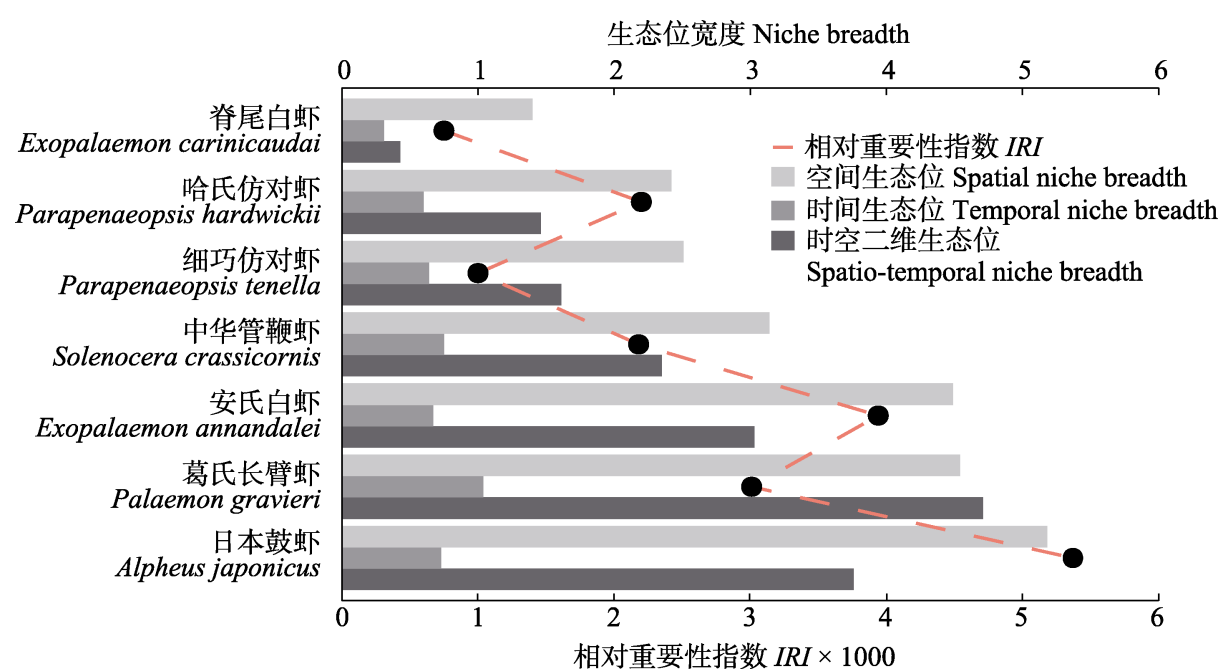

图4 优势种虾类的时间、空间生态位宽度, 时空生态位宽度以及相对重要性指数的比较

Fig. 4 Comparison of temporal niche breadth, spatial niche breadth, spatio-temporal niche breadth and IRI of dominant shrimp species

物种在站位中的出现频率呈显著相关(相关性系数 为 $0.72, P=0.00013)$; 而生态位宽度的计算则考虑 了每个站位(或季节)中某一物种的渔获尾数占比。 由此可知, 某一物种的生物量是否均匀分布于各站 位(或季节)将直接影响其生态位宽度值大小, 而其 $I R I$ 值则不受影响。因此, 生态位宽度值除了能反映 物种对资源的利用能力外, 还能从一定程度上反映 物种是否均匀分布于生境中(徐春燕等, 2012)。这一 结论在此次调查中得到充分的验证: 以渔获虾类的 空间生态位宽度为例 $\left(B_{\mathrm{i}}\right)$, 高生态位宽度组和低生 态位宽度组虾类在各调查站位中的平均出现频率 分别为 $97.91 \%$ 和 $45.63 \%$, 差异明显。

值得注意的是, 相对重要性指数与生态位宽度 均不能反映虾类总生物量的高低。在本次调查中, 长毛明对虾(Fenneropenaeus penicillatus, $401.9 \mathrm{~g} / \mathrm{h}$ ) 和鲜明鼓虾 $(179.6 \mathrm{~g} / \mathrm{h})$ 的相对资源量分别高于脊尾 白虾 $(227.7 \mathrm{~g} / \mathrm{h})$ 和细巧仿对虾 $(103.3 \mathrm{~g} / \mathrm{h})$ 。然而, 长 毛明对虾的IRI值(7.75)远低于脊尾白虾(752.3), 鲜 明鼓虾的IRI值(374.4) 同样也远低于细巧仿对虾 $(1,002.6)$; 且长毛明对虾和鲜明鼓虾的生态位宽度 (分别为 $0.02 、 1.26$ ) 也分别低于脊尾白虾(1.40)和细 巧仿对虾(2.51)。该结果同时也验证了韩东燕等 (2013)提出的有关 “生态位宽度并不能反映物种总 生物量高低”的研究结论。

\section{参考文献}

Bennett JR, Cumming BF, Ginn BK, Smol JP (2010) Broad-scale environmental response and niche conservatism in lacustrine diatom communities. Global Ecology and Biogeography, 19, 724-732.

Chen WF, Peng X, Wang ZH, Xie QL, Chen SB, Ye S, Chen X, Ai WM (2017) Community structure characteristics of fishes in the coastal area of south Zhejiang during autumn and winter. Ocean Development and Management, 34(11), 111-119. (in Chinese with English abstract) [陈伟峰, 彭欣, 汪振华, 谢起浪, 陈少波, 叶深, 陈骁, 艾未明 (2017) 浙南近海秋冬季鱼类群落结构研究. 海洋开发与管理, 34(11), 111-119.]

Ge BM, Bao YX, Zheng X, Cheng HY (2005) The structure of the macrobenthic community and niche analysis at a tidal flat of Lingkun Island, China. Acta Ecologica Sinica, 25, 3037-3043. (in Chinese with English abstract) [葛宝明, 鲍 毅新, 郑祥, 程宏毅 (2005) 灵昆岛潮间带大型底栖动物 群落结构与生态位分析. 生态学报, 25, 3037-3043.]

General Administration of Quality Supervision, Inspection and Qurantine of the People's Republic of China (2007) Specifications for Oceanographic Survey-Part 6: Marine Biological Survey. Standards Press of China, Beijing. (in Chinese) [中华人民共和国国家质量监督检验检疫总局 (2007) 海 洋调查规范 第6部分: 海洋生物调查. 中国标准出版社, 北京.]

Guo TY, Xu RM (2003) Study on time niche among the ectoparasites of the rodent in Dongling Mountain in Beijing, China. Chinese Journal of Vector Biology and Control, 14, 30-32. (in Chinese with English abstract) [郭天宇, 许荣满 (2003) 北京东灵山地区鼠类体外寄生虫时间生态位的研 究. 中国媒介生物学及控制杂志, 14, 30-32.]

Han DY, Xue Y, Ji YP, Xu BD, Liu H, Ma QY (2013) Trophic and spatial niche of five gobiid fishes in Jiaozhou Bay. Journal of Fishery Sciences of China, 20, 148-156. (in Chinese with English abstract) [韩东燕, 薛荣, 纪毓鹏, 徐宾 铎, 刘贺, 麻秋云 (2013) 胶州湾5种虾虎鱼类的营养和 空间生态位. 中国水产科学, 20, 148-156.]

He ZT, Xu KD, Xue LJ, Song HT (2009) Estimation of 
biological parameter, biomass, and yield for Palaemon gravieri in the northern area of East China Sea. Journal of Zhejiang Ocean University (Natural Science), 28, 286-291. (in Chinese with English abstract) [贺舟挺, 徐开达, 薛利 建, 宋海棠 (2009) 东海北部葛氏长臂虾生长死亡参数 及资源量、渔获量的分析. 浙江海洋学院学报(自然科学 版), 28, 286-291.]

He ZT, Zhang HL, Xu KD, Liu ZF (2012) Diversity and distribution of benthic organisms in rocky intertidal zone of Jiushan Islands Nature Reserve, China. Fishery Information \& Strategy, 27(3), 151-156. (in Chinese with English abstract) [贺舟挺, 张洪亮, 徐开达, 刘子藩 (2012) 非山列 岛自然保护区岩相潮间带底栖生物多样性与分布. 渔业 信息与战略, 27(3), 151-156.]

Li DZ, Shi Q, Zang RG, Wang XP, Sheng LJ, Zhu ZL, Wang CA (2006) Models for niche breadth and niche overlap of species or populations, China. Scientia Silvae Sinicae, 42(7), 95-103. (in Chinese with English abstract) [李德志, 石强, 蔵润国, 王绪平, 盛丽娟, 朱志玲, 王长爱 (2006) 物种 或种群生态位宽度与生态位重叠的计测模型. 林业科学, 42(7), 95-103.]

Li R, Li SC, Tian RJ (2007) Structure and niche of spider community in vegetable field, China. Acta Arachnologica Sinica, 16, 116-120. (in Chinese with English abstract) [李 锐, 李生才, 田瑞钧 (2007) 菜田蜘蛛群落组成及生态位 分析. 蛛形学报, 16, 116-120.]

Liu RY (1963) Zoogeographical studies on the macrurous crustacean fauna of the Yellow Sea and the East China Sea. Oceanologia et Limnologia Sinica, 5(3), 230-244. (in Chinese with English abstract) [刘瑞玉 (1963) 黄、东海虾类 动物地理学研究. 海洋与湖沼, 5(3), 230-244.]

Liu RY (2008) Checklist of Marine Biota of China Seas. Science Press, Beijing. (in Chinese) [刘瑞玉 (2008) 中国海洋 生物名录. 科学出版社, 北京.]

Lu ZH, Xu HX, Xue LJ, Zhu WB (2015) A study on seasonal changes of the shrimp communities in the middle East China Sea. Journal of Zhejiang Ocean University (Natural Science), 34, 497-504. (in Chinese with English abstract) [卢占 晖, 徐汉祥, 薛利建, 朱文斌 (2015) 东海中部虾类群落 的季节变化研究. 浙江海洋学院学报(自然科学版), 34, 497-504.]

Lu ZH, Xue LJ, Zhang L, Xu KD, Zhang YZ (2013) Assessment of shrimp biomass in continental shelf waters of the East China Sea, China. Acta Hydrobiologica Sinica, 37, 855-862. (in Chinese with English abstract) [卢占晖, 薛利 建, 张龙, 徐开达, 张亚洲 (2013) 东海大陆架虾类资源 量评估. 水生生物学报, 37, 855-862.]

Lu ZH, Zhou YD, Zhu WB, Xu KD (2018) Spatial niches analysis of dominant shrimp species in the coastal area of Zhejiang Province. Acta Oceanologica Sinica, 40(2), 77-86. (in Chinese with English abstract) [卢占晖, 周永东, 朱文 斌, 徐开达 (2018) 浙江沿岸海域虾类优势种空间生态 位分析. 海洋学报, 40(2), 77-86.]

Magurran AE (1988) Ecological Diversity and Its Measurement. Princeton University Press, New Jersey.

May RM (1975) Some notes on estimating the competition matrix. Ecology, 56, 737-741.

Peng SY, Li XZ, Wang HF, Zhang BL (2015) Niche analysis of dominant species of macrozoobenthic community in the southern Yellow Sea in spring. Acta Ecologica Sinica, 35, 1917-1928. (in Chinese with English abstract) [彭松耀, 李 新正, 王洪法, 张宝琳 (2015) 南黄海春季大型底栖动物 优势种生态位. 生态学报, 35, 1917-1928.]

Pianka ER (1973) The structure of lizard communities. Annual Review of Ecology \& Systematics, 4(41), 53-74.

Pinkas L, Oliphant MS, Iverson ILK (1971) Food habits of albacore, bluefin tuna, and bonito in California waters. Fish Bulletin, 152, 1-105.

Qi HM, Sun Y, Xu ZL, Sun LF, Gao Q, Que JL, Tian W (2013) Shrimp community structure and its influential factors in the Jiaojiang River estuary during spring and autumn. Chinese Journal of Applied Ecology, 24, 3546-3552. (in Chinese with English abstract) [齐海明, 孙岳, 徐兆礼, 孙鲁峰, 高 倩, 阙江龙, 田伟 (2013) 椒江口海域春秋季虾类群落结 构及其影响因素. 应用生态学报, 24, 3546-3552.]

R Core Team (2016) R: A Language and Environment for Statistical Computing. R Foundation for Statistical Computing, Vienna.

Shi YH, Zhang GY, Liu JZ, Zhu YZ, Zhu XD (2009) Growth and reproductive characteristics of Exopalaemon carinicauda bred in different months. Fisheries Science \& Technology Information, 36(3), 131-136. (in Chinese with English abstract) [施永海, 张根玉, 刘建忠, 朱雅珠, 朱晓 东 (2009) 不同月份出生的脊尾白虾之生长及生殖特性. 水产科技情报, 36(3), 131-136.]

Song HT (2002) The ecological colony and fauna characteristics of East China Sea shrimp. Studia Marina Sinica, 44, 124-133. (in Chinese with English abstract) [宋海棠 (2002) 东海虾类的生态群落与区系特征. 海洋科学集刊, 44, 124-133.]

Song HT, Yu CG, Xue LJ (2009) Study on the biomass distribution and growth property of Parapenaeopsis hardwickii in the East China Sea. Acta Hydrobiologica Sinica, 33, 15-21. (in Chinese with English abstract) [宋海棠, 俞存根, 薛利 建 (2009) 东海哈氏仿对虾的数量分布和生长特性研究. 水生生物学报, 33, 15-21.]

Song HT, Yu CG, Xue LJ (2012) Fishery Biology of Commercial Shrimp and Crab Species in the East China Sea. China Ocean Press, Beijing. (in Chinese) [宋海棠, 俞存根, 薛利 建 (2012) 东海经济虾蟹类渔业生物学. 海洋出版社, 北 京.]

Sun LH, Li XF, Wang YN, Jiao HF, Shen YQ, Zhang SF, He J (2014) The species and distribution of macrobenthos in the Nanjiushan Sea area. Ecological Science, 33(1), 32-37. (in Chinese with English abstract) [孙立辉, 李祥付, 王一农, 焦海峰, 沈叶青, 张树枫, 何京 (2014) 南非山周围海域 大型底栖动物种类与分布. 生态科学, 33(1), 32-37.]

Tang FH, Wu YM, Fan W, Shen XQ, Wang YL (2010) Preliminary discussion on phytoplankton distribution and its relation to the runoff in the Yangtze River estuary. Ecology and Environment Sciences, 19, 2934-2940. (in Chinese with English abstract) [唐峰华, 伍玉梅, 樊伟, 沈新强, 王云龙 
(2010) 长江口浮游植物分布情况及与径流关系的初步探 讨. 生态环境学报, 19, 2934-2940.]

Tang YB, Liao YB, Shou L, Zeng JN, Gao AG, Chen QZ (2016) Intertidal zone of Nanji Islands is a niche for dominant species of the microbenthic community. Acta Ecologica Sinica, 36, 489-498. (in Chinese with English abstract) [汤 雁滨, 廖一波, 寿鹿, 曾江宁, 高爱根, 陈全震 (2016) 南鹿列岛潮间带大型底栖动物群落优势种生态位. 生态 学报, 36, 489-498.]

Tian MC, Sun BL, Yang JM (1993) An analysis of ichthyologic fauna in Bohai Sea. Studia Marina Sinica, 34, 157-167. (in Chinese) [田明诚, 孙宝龄, 杨纪明 (1993) 渤海鱼类 区系分析. 海洋科学集刊, 34, 157-167.]

Wang YH, Wu H, Zhu JR, Shen J (2015) Numerical simulation on the phytoplankton dynamics in Changjiang estuary and Min-Zhe coastal waters. Journal of East China Normal University (Natural Science), (4), 97-109. (in Chinese with English abstract) [王一鹤, 吴辉, 朱建荣, 沈健 (2015) 长 江口及闽浙海域浮游植物动力学模型研究. 华东师范大 学学报(自然科学版), (4), 97-109.]

Wathne JA, Haug T, Lydersen C (2000) Prey preference and niche overlap of ringed seals Phoca hispida and harp seals P. groenlandica in the Barents Sea. Marine Ecology Progress, 194, 233-239.

Wu CW, Wang WH (1995) A study of biology and ecological distribution of Exopalaemon annandalei in Hangzhou Bay. Journal of Zhejiang Ocean University (Natural Science), 12, 21-31. (in Chinese) [吴常文, 王伟洪 (1995) 杭州湾海域 安氏白虾生物学与生态分布的研究. 浙江水产学院学报 (自然科学版), 12, 21-31.]

Xia LJ, Chen WD, Zheng J, Cai HC, Wu EW, Bi SY, Xie X, Yu CG (2016) Species composition and quantitative distribution of shrimp in the Nanji Islands Marine Conservation Area. Journal of Fishery Sciences of China, 23, 648-660. (in Chinese with English abstract) [夏陆军, 陈万东, 郑基, 蔡 厚才, 伍尔魏, 毕耛瑶, 谢旭, 俞存根 (2016) 南鹿列岛 海洋自然保护区的虾类种类组成和数量分布. 中国水产 科学, 23, 648-660.]

Xie X, Yu CG, Zheng J, Xia LJ, Bi SY, Zhang P, Deng XY, Miao L (2017) Community structure and relationship with environment in shrimp in Pishan waters at Yushan Fishing Ground. Journal of Dalian Ocean University, 32, 713-723. (in Chinese with English abstract) [谢旭, 俞存根, 郑基, 夏陆军, 毕耤瑶, 张平, 邓小艳, 苗露 (2017) 鱼山渔场 披山海域虾类群落结构及其与环境因子的关系. 大连海 洋大学学报, 32, 713-723.]

Xu CY, Yu QJ, Xu FJ, Hu XQ, You WH (2012) Niche analysis of phytoplankton's dominant species in Dianshan Lake of East China. Chinese Journal of Applied Ecology, 23, 2550-2558. (in Chinese with English abstract) [徐春燕, 俞 秋佳, 徐风洁, 胡雪芹, 由文辉 (2012) 淀山湖浮游植物 优势种生态位. 应用生态学报, 23, 2550-2558.]

Xu JP, Yang SY (1993) A discussion of environmental resources and its development in Sanmen Bay. Ocean Development and Management, (2), 14-16. (in Chinese) [许建平,
杨士英 (1993) 三门湾环境资源及其综合开发探讨. 海 洋与海岸带开发, (2), 14-16.]

Xu JY (1990) A preliminary research of reproduction of Exopalaemon annandalei in Yueqing Bay, Zhejiang. Chinese Journal of Zoology, 25(6), 3-7. (in Chinese) [徐君义 (1990) 浙江乐清湾脊尾白虾的繁殖和世代的初步研究. 动物学杂志, 25(6), 3-7.]

Xu XQ, Zeng JN, Chen QZ, Liu JJ, Du P, Wang GZ (2013) Spatial niches of dominant zooplankton species in Sanmen Bay, Zhejiang Province of East China. Chinese Journal of Applied Ecology, 24, 818-824. (in Chinese with English abstract) [徐晓群, 曾江宁, 陈全震, 刘晶晶, 杜萍, 王桂忠 (2013) 浙江三门湾浮游动物优势种空间生态位. 应用生 态学报, 24, 818-824.]

Xu ZG, He Y, Yan BX, Song CC (2007) Niche characteristics of typical marsh wetland plant populations in Sanjiang Plain. Chinese Journal of Applied Ecology, 18, 783-787. (in Chinese with English abstract) [徐治国, 何岩, 间百兴, 宋 长春 (2007) 三江平原典型沼泽湿地植物种群的生态位. 应用生态学报, 18, 783-787.]

Xue LJ, He ZT, Xu KD, Song HT (2009) Population dynamics and estimation of sustainable yield for Solenocera crassicornis in the East China Sea. Journal of Fujian Fisheries, (4), 48-54. (in Chinese with English abstract) [薛利建, 贺舟挺, 徐开达, 宋海棠 (2009) 东海中华管鞭虾种群动 态及持续渔获量分析. 福建水产, (4), 48-54.]

Yu ZH, Jin XS, Li XS (2010) Analysis of ecological niche for major fish species in the central and southern Yellow Sea. Marine Fisheries Research, 31(6), 1-8. (in Chinese with English abstract) [于振海, 金显仕, 李显森 (2010) 黄海 中南部主要鱼种的生态位分析. 渔业科学进展, 31(6), 1-8.]

Zhang GL, Zhang JT (2002) Niche analysis of dominant species in Shenweigou of Guandi Mountain. Journal of Wuhan Botanical Research, 20, 203-208. (in Chinese with English abstract) [张桂莲, 张金屯 (2002) 关帝山神尾沟优势种 生态位分析. 武汉植物学研究, 20, 203-208.]

Zhang HL, Xu KD, He ZT, Liu ZF, Zhou YD (2008) Analysis on the condition of fishery resources in the sea area around the Jiushan Archipelago. Marine Fisheries, 30, 105-113. (in Chinese with English abstract) [张洪亮, 徐开达, 贺舟挺, 刘子藩, 周永东 (2008) 非山列岛附近海域渔业资源分 析. 海洋渔业, 30, 105-113.]

Zhang JT (2011) Quantitative Ecology, 2nd edn. Science Press, Beijing. [张金屯 (2011) 数量生态学, 第2版. 科学出版 社, 北京.]

Zhang YZ, He ZT (2013) Analysis on the fishery resources in the sea area of Jiushan Archipelago Oceanic Ecology Reserve during spring and summer. Journal of Zhejiang Ocean University (Natural Science), 32, 292-298. (in Chinese with English abstract) [张亚洲, 贺舟挺 (2013) 春、夏季非山列 岛海洋生态自然保护区海域渔业资源分析. 浙江海洋学 院学报(自然科学版), 32, 292-298.]

(责任编委: 李新正 责任编辑: 间文杰) 